\title{
Distributed Uplink Macro Diversity for Cooperating Base Stations
}

\author{
Laëtitia Falconetti \\ Ericsson Research \\ Aachen, Germany
}

\author{
Christian Hoymann \\ Ericsson Research \\ Aachen, Germany
}

\author{
Rohit Gupta \\ University of Washington \\ Seattle, Washington, USA
}

\begin{abstract}
Nowadays cellular systems operate with frequency reuse one, where adjacent cells use the same frequency band. Use Equipments (UEs) located at cell edge are mostly affected by the resulting co-channel interference. In addition, cell edge UEs suffer from their weak carrier signal strength.

This paper proposes a new method to increase the performance of cell edge UEs by means of information exchange between Base Stations (BSs). A BS serving a cell edge UE requests support from a co-channel BS. The supporting BS transfers demodulated or decoded bits received from the cell edge UE back to the serving BS. The serving BS then combines the information. The concept of cooperative BSs described in this paper is based on a requestresponse mechanism and does not require a central control node. Performance evaluation by means of simulation shows the capability of BS cooperation applied to 3GPP Long-Term Evolution (LTE) in terms of user throughput and emphasizes the trade-off in terms of increased backhaul requirement due to BS-BS communication.
\end{abstract}

\section{INTRODUCTION}

In cellular systems with tight frequency reuse, UEs located in nearby co-channel cells may be scheduled on the same physical resources. Their simultaneous transmissions create co-channel interference which reduces Signal to Interference plus Noise Ratio (SINR) and therefore limits capacity.

In conventional cellular systems, co-channel interference is reduced by Radio Resource Management (RRM) such as power control, loose frequency reuse, spreading code assignments, and inter-cell interference coordination. Uplink (UL) co-channel interference can also be mitigated at the BS receiver by means of multi-antenna processing, e.g., Interference Rejection Combining (IRC) receivers [1]. More advanced receiver algorithm such as Interference Cancelation (IC) can remove interference if the interfering streams are known at the receiver [2], which is usually not the case for co-channel interference.

Leveraging antennas of several cells allows multi-user detection across cell borders leading to improved link quality. Such cooperation across cells is most often modeled as a single super-BS with remote antennas in several cells [3], [4]. This centralized approach mitigates interference between cells of the same super-BS, but interference between cells of different super-BSs remains. BS cooperation with distributed control as in [5] shows a comparable performance gain without border

This work has been partly funded by the German Federal Ministry of Education and Research under the grant 01BU0634 (EASY-C). The authors acknowledge the excellent cooperation within the project. effects, however, it requires high-capacity BS-BS interfaces whose traffic is carried by the backhaul network.

This paper investigates cooperative BSs with reduced backhaul capacity requirements. The proposed method enables BSs to demodulate (or decode) the signal received from a UE associated to a nearby co-channel cell. The demodulated (or decoded) signal is transferred to the serving BS, which combines that information with its own signal.

The approach is similar to soft (or softer) handover, as specified for 3GPP UMTS, where information received in different cells is combined in the Radio Network Controller. In order to allow BS cooperation without centralized coordination the described approach is based on a request-response mechanism with distributed control. Therefore, UL cooperation of BSs can be integrated in the 3GPP logical LTE/SAE architecture. The approach is backward compatible and can be seen as a potential evolution.

The paper is organized as follows. Section II briefly summarizes relevant features of LTE and the corresponding core network architecture named System Architecture Evolution (SAE). The new concept of UL cooperation between BSs is introduced in section III and the impact on BS algorithms is discussed in section IV. Section V presents performance results of BS cooperation applied to LTE. A conclusion is drawn in section VI.

\section{3GPP LONG-TERM EVOLUTION}

\section{A. LTE/SAE logical architecture}

3GPP's core network (SAE) and the radio access (LTE) are evolving in parallel [1]. The resulting flat architecture is composed of only two logical nodes in the user plane (UP): the eNodeB and the Serving Gateway (S-GW), see Fig. 1. The S$\mathrm{GW}$ executes packet filtering, classification and it provides the connection to the Internet or to other telephony network. An eNodeB provides the LTE radio access. Like in the UP, only two nodes are involved in the control plane (CP): the eNodeB and the Mobility Management Entity (MME). The MME handles core network control functions, such as attach/detach handling, mobility functions, bearer management, and security. eNodeBs are connected to the core network using the IP-based interface S1. The logical interface between eNodeBs, i.e., the IP-based X2 interface supports loss-less mobility and multicell RRM. 


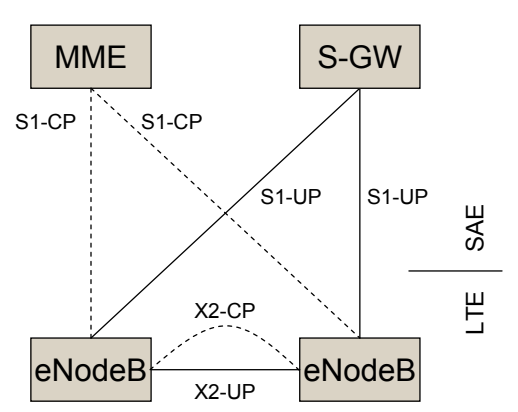

Fig. 1. LTE/SAE logical architecture

\section{B. LTE physical layer}

LTE supports flexible carrier bandwidths up to $20 \mathrm{MHz}$. Different advanced multi antenna solutions exploiting high SINR and low SINR regions are key components. Up to 4 receive (Rx) antenna ports are available at the BS while UEs use a single antenna port for transmission.

LTE uses Orthogonal Frequency Division Multiple Access (OFDMA) for the downlink (DL). OFDMA divides the broad carrier bandwidth in a large number of narrow subcarriers that are modulated and allocated independently among users. The Inverse Fast Fourier Transform of the modulated subcarriers results in an OFDM symbol. Physical resources can be seen as a time-frequency grid which enables a channel-dependent allocation in time and frequency domains. In LTE a Resource Block (RB) is the smallest unit that can be allocated. It is composed of 14 OFDM symbols and 12 subcarriers.

In UL, LTE uses a pre-coded version of OFDM called Single Carrier FDMA (SC-FDMA), which groups allocated resources and pre-codes them with a Fast Fourier Transform. The resulting transmission has Single Carrier (SC)-like characteristics.

\section{LTE medium access control layer}

The Medium Access Control (MAC) layer performs multiplexing, Hybrid ARQ (HARQ), and scheduling. The scheduler entity of an eNodeB allocates uplink and downlink resources while the entity in the UE just acts according to the assigned grants. MAC scheduling also controls HARQ retransmissions and adapts modulation schemes and coding rates.

\section{UL COOPERATION OF BASE STATIONS}

According to LTE operations, each UE is associated to one serving BS which controls UE transmissions, e.g., resource allocation, Modulation and Coding Scheme (MCS) selection. The newly proposed scheme enables the serving BS to request cooperation from one or more co-channel BSs for certain UEs. The exchanged information can either be uncoded bits or soft values of coded bits.

\section{A. Coded bit exchange}

Fig. 2 shows a message sequence chart of BS cooperation based on the exchange of coded bits. After scheduling a celledge UE on certain RBs, the serving BS requests coded bits

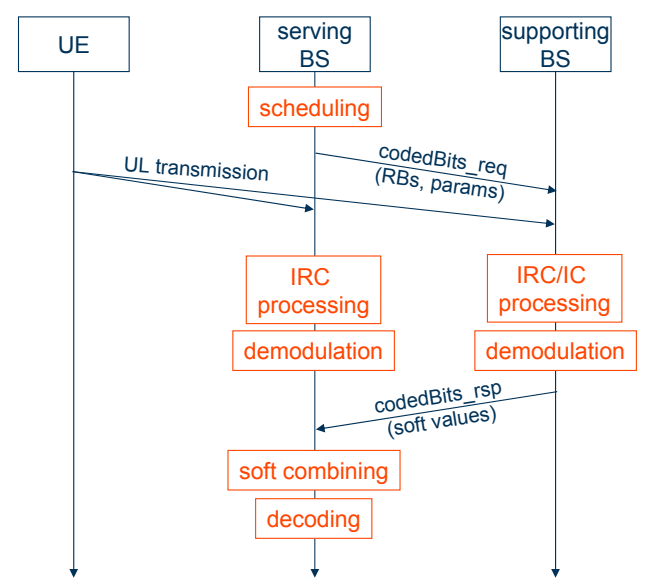

Fig. 2. Message sequence chart for requesting soft values of coded bits from supporting BS

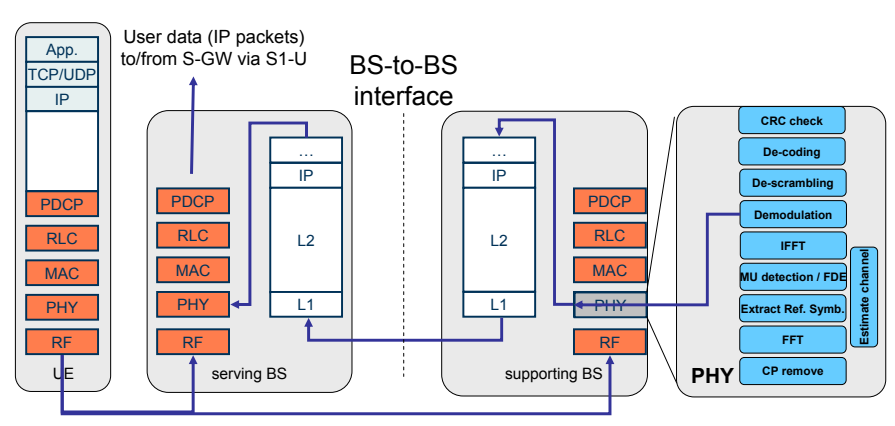

Fig. 3. User plane protocol stack of cooperating LTE BSs in case of soft bit exchange

from an adjacent co-channel BS, called supporting BS. The request message contains information about the allocated RBs and additional transmit (Tx) parameters, such as modulation scheme, UE specific reference symbols etc. The supporting BS should detect and demodulate the requested UE signal. It can apply receiver algorithms that mitigate interference, e.g., IRC. In case of an overlapping transmission, the supporting BS can first detect and decode the signal from its own UE, which is most probably the strongest signal. According to the IC principle, it can then cancel the signal of its own UE and detect the requested signal of the adjacent co-channel UE.

After demodulation, the supporting BS transfers the quantized soft values of the coded bits back to the serving BS. The serving BS combines the soft values of the supporting BS with the soft values of its own coded bits. Chase combining, which is already used to combine HARQ retransmissions can be applied. Finally, decoding takes place at the serving BS.

An example LTE UP protocol stack of cooperating BSs is shown in Fig. 3. The right hand side of the figure focusses on the LTE BS Physical Layer (PHY) layer. The supporting BS extracts coded bits from its demodulation module and transfers them to the serving BS via the BS-BS interface. This BS-BS interface can be any interface that fulfils the capacity and delay requirements, see sections IV-A and V-C. 


\section{B. Uncoded bit exchange}

In order to reduce the backhaul requirement, the serving BS can request decoded (hard) bits from a supporting BS. Basically a request-response message exchange similar to Fig. 2 takes place. As additional parameter, the request message contains the coding scheme used by the UE. The supporting BS detects, demodulates and decodes the signal. If the signal is correctly decoded, i.e. when the CRC is successful, the supporting BS transfers the decoded data back to the serving BS. After receiving the response message, the serving BS performs selection combining: the serving BS uses the hard bits sent by the supporting BSs when it cannot decode the data itself.

\section{Algorithms AFFECTED By BS COOPERATION}

To enable efficient BS cooperation some existing BS functionalities have to be adjusted.

\section{A. Hybrid $A R Q$}

LTE specifies that the HARQ feedback is sent three Transmission Time Intervals (TTIs) after the data transmission. In case of BS cooperation the serving BS cannot transmit a reasonable ACK/NACK feedback before it has received and combined the coded (uncoded) bits from the supporting BSs with its own coded (uncoded) bits. Thus, either the process of BS cooperation including signal processing and coded (uncoded) bit exchange can be finished in time or the HARQ mechanism has to be adapted to allow for longer feedback delays.

\section{B. Selection of UEs and BSs involved in cooperation}

The above described BS cooperation modes do not involve central control. If the serving BS needs support for particular UEs, it sends a request to certain co-channel BSs. The choice of supporting BSs is decisive due to its high impact on performance. For example, BS cooperation with hard bit exchange is not much beneficial for a UE located in the cell center, as the neighbor BS receiving its signal with a relatively low power will in most cases not be able to decode it correctly. Thus, cell edge users receiving signals from the serving BS and the supporting BSs with comparable strength are more appropriate to explore the potential of BS cooperation.

Cell edge UEs usually carry out and report signal strength measurements for mobility purposes. These reports can be used by the serving BS to select a set of potential supporting $\mathrm{BSs}$ that will receive a cooperation request. However, the supporting BS can reject some cooperation requests. Indeed, the number of co-channel UEs supported by a BS should be limited by the number of BS Rx antennas to ensure an efficient multi-user detection.

\section{Link adaptation algorithms}

Link adaptation is necessary to overcome varying channel conditions and interference levels. The MCS and the Tx power of an UL transmission is adapted to the SINR experienced at the BS. A robust MCS and a high Tx power can cope with unfavorable channel conditions. If the $\mathrm{Rx}$ signal quality is good, the Tx power can be decreased to reduce interference caused to co-channel UEs and/or a more aggressive MCS can be used to improve the achieved throughput.

BS cooperation increases UL Rx signal quality by leveraging the multiple channels from the UE to a plurality of BSs. The improved Rx signal quality can be used as input to the MCS and Tx power selection algorithm. To obtain an estimate of the improved SINR, the serving BS could request the supporting BS to report a SINR measurement of the signal it receives from the supported UE. The serving BS can then compute the increased SINR due to BS cooperation.

\section{Interference Cancelation}

The supporting BS should be able to separate signals of supported UEs and signals of its own UEs scheduled on the same RBs. Receiver algorithms mitigating interference such as IRC can be used at the BS. IC receivers can also be applied at the supporting BS to cancel the known signal of its own UE, which is perceived as interference by the serving BS's UE [6].

When exchanging uncoded bit the supporting BS knows the signal of the supported UE, which could then be canceled as well. In case of coded bit exchange, the signal received from supported UE is not decoded and error checked. Thus, interference caused by such UEs cannot be canceled.

\section{Performance Evaluation}

\section{A. Simulation environment}

BS cooperation has been evaluated by simulating a $1 \times 4$ LTE UL transmission in a fully loaded $10 \mathrm{MHz}$ FDD LTE network consisting of 7 sites with three sectors (cells) per site. The inter-site distance is $500 \mathrm{~m}$. Each cell has 10 users in average and operates at a carrier frequency of 2 $\mathrm{GHz}$. The evaluation assumptions are essentially based on the NGMN recommendations [7]. The channel model used for evaluation is the urban scenario outlined in [8] with a user velocity of $3 \mathrm{~km} / \mathrm{h}$. In each cell, a channel dependent scheduler allocates equal number of RBs to users according to their experienced channel conditions. Channel quality is measured on the sounding signal which is transmitted every $20 \mathrm{~ms}$. A conventional open loop power control is applied [1]. QPSK, 16QAM and 64QAM modulation schemes are available. The increased SINR due to BS cooperation is computed in every TTI and is used for link adaptation after a processing delay of another TTI (1ms). A link-to-system interface based on mutual information is used to map the packet SINR to the corresponding packet error probability [9].

A parameter named cooperation range determines which UEs are served under cooperation. Only UEs receiving signals from co-channel BSs within a certain cooperation range below the signal received from the serving BS are chosen for cooperation. The corresponding BSs are selected as candidate supporting BSs for the considered UE. A parameter limits the maximum number of supporting BSs per UE. In this scenario, a BS can serve simultaneously up to 4 (own or supported) UEs on the same RBs, see IV-B. 


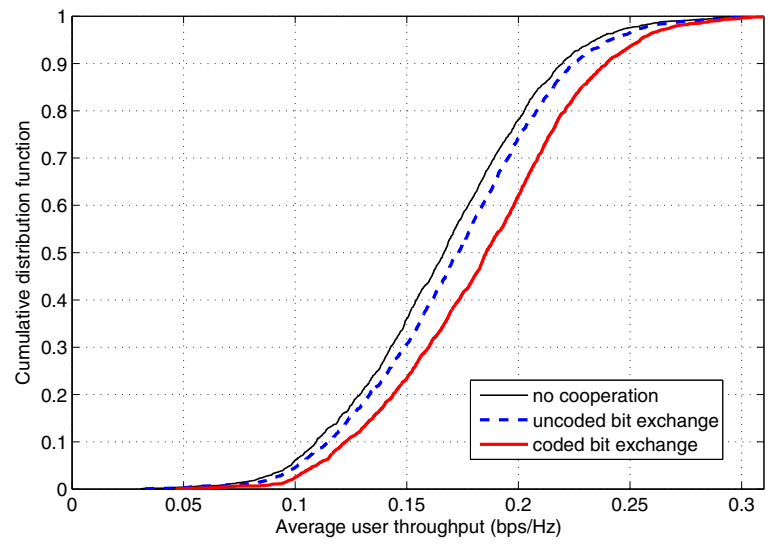

Fig. 4. CDF of average user throughput for different BS cooperation modes (IRC receiver, coded bit exchange: max. 2 supporting cells, cooperation range of $10 \mathrm{~dB}$ )

TABLE I

AVERAGE AND 5\%-PERCENTILE USER THROUGHPUT (IRC RECEIVER, $\mathrm{UB}=\mathrm{UNCODED} \mathrm{BIT}, \mathrm{CB}=\mathrm{CODED} \mathrm{BIT})$

\begin{tabular}{|l|r|r|r|r|}
\hline Cooperation mode & \multicolumn{2}{|c|}{$\begin{array}{c}\text { Av. user } \\
\text { throughput }\end{array}$} & \multicolumn{2}{c|}{$\begin{array}{c}\text { 5\%-percentile } \\
\text { user throughput }\end{array}$} \\
\hline & {$\left[{ }^{b p s} / \mathrm{Hz}\right]$} & {$[\%]$} & {$\left[{ }^{b p s} / \mathrm{Hz}\right]$} & {$[\%]$} \\
\hline No cooperation & 0.166 & & 0.098 & \\
\hline UB, 2 supp. cells, 10dB & 0.172 & +3.6 & 0.102 & +4.1 \\
\hline $\mathrm{CB}, 2$ supp. cells, 3dB & 0.175 & +5.4 & 0.104 & +6.1 \\
\hline $\mathrm{CB}, 1$ supp. cells, 10dB & 0.180 & +8.4 & 0.107 & +9.2 \\
\hline $\mathrm{CB}, 2$ supp. cells, 10dB & 0.184 & +10.8 & 0.109 & +11.2 \\
\hline $\mathrm{CB}, 21$ supp. cells, $\infty$ & 0.188 & +13.3 & 0.112 & +14.3 \\
\hline
\end{tabular}

\section{B. Performance increase}

Fig. 4 represents the Cumulative Distribution Function (CDF) of the average user throughput when exchanging coded and uncoded bits. As shown in Tab. I, the coded bit exchange mode outperforms the uncoded bit exchange mode in terms of average and cell edge user throughput. This comes from the fact that by combining soft values of coded bits received from several supporting BSs with its own, the serving BS aggregates the information of the UE signal received through several channels. In case of uncoded bit exchange, the serving BS can only select between using the bit stream from the supporting BS or using its own bit stream.

Cooperation by means of uncoded bit exchange is rather insensitive to the variation of the cooperation range and of the maximum number of cooperating cells. Increasing the maximum number of supporting cells, e.g., from 2 to 5, does not affect the average user throughput. Increasing the cooperation range, e.g., from 10 to $40 \mathrm{~dB}$ has no influence, too. The corresponding CDFs match. This is due to the larger pathloss from the UE to the additional supporting cell, which is most probably not able to decode the UE's signal successfully. Note that, the term supporting cell used here refers to the part of the supporting BS that serves the particular cell.

Fig. 5 shows the CDF of the average user throughput when exchanging coded bit for different cooperation ranges

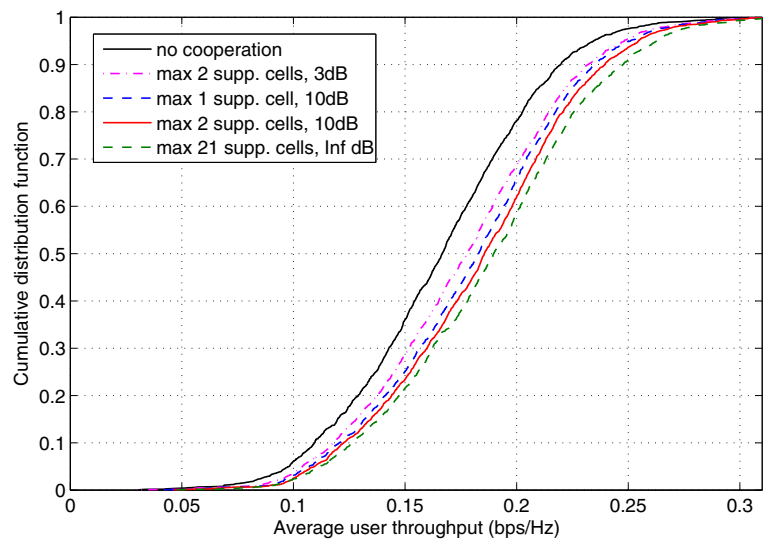

Fig. 5. CDF of average user throughput for different cooperation parameters (IRC receiver, coded bit exchange)

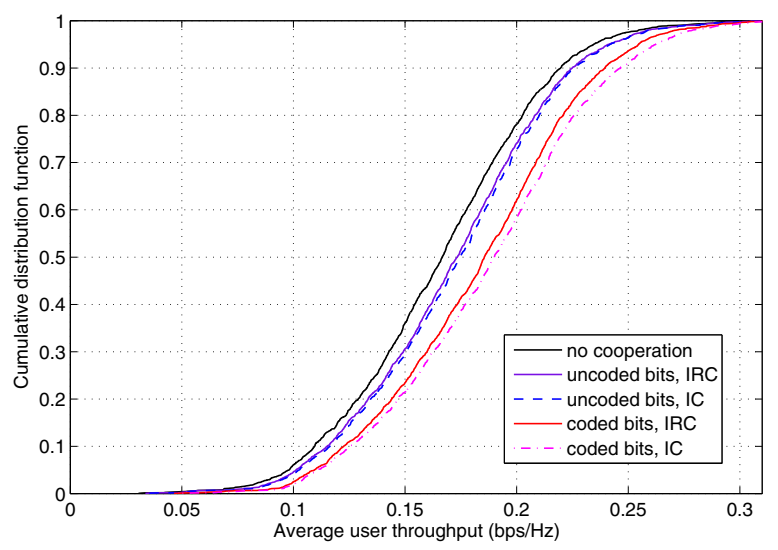

Fig. 6. CDF of average user throughput for different BS cooperation modes and receiver algorithms (max. 2 supporting cells and 10dB cooperation range)

and different values of the maximum number of supporting cells per UE. At a constant cooperation range of $10 \mathrm{~dB}$, performance increases moderately when increasing the number of supporting cells, see Tab. I. With soft combining each additional cell increases the SINR of the UE's Rx signal and therewith the probability of correct decoding. However, the additional supporting cell is farther away and its soft values do not contain as much information as the soft values of the serving BS or a closer supporting BS.

By increasing the cooperation range from 3 to $10 \mathrm{~dB}$, at a constant maximum number of supporting cells, the number of UEs eligible for cooperation rises and the mean user throughput improves accordingly, see Tab. I.

Fig. 5 also shows the maximum performance when exchanging coded bit: all 21 cells of the scenario cooperate for every UE. However, a cooperation approach with a limited cooperation range, e.g. $10 \mathrm{~dB}$, and a small number of supporting cells, e.g. up to 2 , yields a mean user throughput close to the maximum achievable.

Fig. 6 shows the influence of two different receiver algo- 


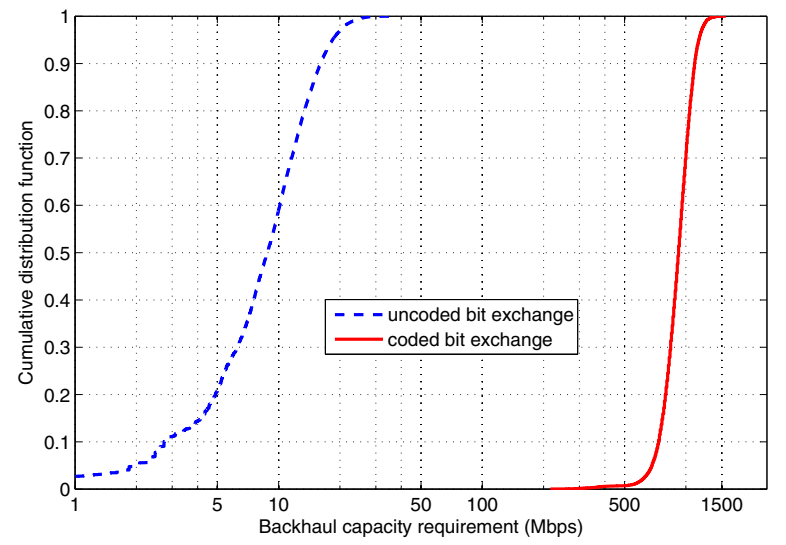

Fig. 7. CDF of backhaul capacity requirement per site for different BS cooperation modes (IRC receiver, uncoded bit: max. 1 supporting cell and $3 \mathrm{~dB}$ range, coded bit: max. 2 supporting cells and $10 \mathrm{~dB}$ range)

rithms applied at the supporting BS: IRC, where interference caused by co-channel UEs is mitigated, and IC, where in addition the supporting BS cancels the interference caused by its own UE. The CDF of the user throughput shows a modest improvement with IC compared to IRC with both coded and uncoded bits exchange. Compared to IRC, IC increases the performance by $0.6 \%$ with uncoded bit exchange and by about $2 \%$ with coded bit exchange.

Canceling interference caused by the supporting BS's own UE removes the strongest interferer and significantly improves the quality of the received signal at the supporting BS. However, the final performance is determined by the combination of the information received at the, most often dominating, serving BS and the supporting BSs. Since IC only improves the signal quality at the supporting BSs, gains are only marginal.

\section{Backhaul requirement}

By means of system level simulations, the required backhaul capacity is measured for each three-sectored site as the sum of the input and output traffic generated by the exchange of uncoded bits or soft values of coded bits with BSs at other sites. Here, one soft value of a coded bit is assumed to be quantized with 5 bits. Fig. 7 shows the resulting backhaul capacity requirement for both cooperation modes. For each mode, the most appropriate parameter set is used: a $3 \mathrm{~dB}$ range with up to 1 supp. cell for uncoded bit exchange and a $10 \mathrm{~dB}$ range with up to 2 supp. cells for coded bit exchange.

BS cooperation based on uncoded bit exchange results in an average backhaul traffic of $9 \mathrm{Mbps}$. In about $3 \%$ of the TTIs less than $1 \mathrm{Mbps}$ is generated, reflecting the fact that a supporting BS, which cannot decode the requested user data, does not send a cooperation response. Exchanging coded bits, which shows larger gain, requires a higher average backhaul capacity of $900 \mathrm{Mbps}$. As expected there is a tradeoff between (cell edge) user throughput improvement and backhaul capacity requirement. A new metric quantifies the backhaul capacity required to increase the mean cell throughput by $1 \%$. Table
TABLE II

REQUIRED BACKHAUL CAPACITY PER INCREASED CELL THROUGHPUT (IRC RECEIVER, UB = UNCODED BIT, $\mathrm{CB}=$ CODED BIT $)$

\begin{tabular}{|c|c|}
\hline Cooperation mode & $\begin{array}{c}\text { Required backhaul capacity [Mbps] } \\
\text { for } 1 \% \text { increase in cell throughput }\end{array}$ \\
\hline UB, 1 supp. cell, 3dB & 2.5 \\
\hline CB, 2 supp. cells, 10dB & 84 \\
\hline
\end{tabular}

II shows that exchanging uncoded bits is more efficient from a backhaul perspective. However, exchanging soft values of coded bits is more effective since it reaches higher absolute gains.

\section{CONCLUSION}

BS cooperation allows increasing the received signal quality of UEs by leveraging information received at several BSs. Especially cell edge UEs benefit. Supporting BSs generate coded or uncoded bits of the received UE signal and transfer them to the serving BS which combines them with its own bits. The proposed scheme allows requesting cooperation for certain RBs of certain UEs, and thus achieves an efficient use of backhaul capacity. Due to its distributed nature, the cooperation scheme does not require a central controller.

Performance evaluation showed the improvement in cell edge user throughput achieved with BS cooperation applied to LTE: in the considered scenario the cell edge user throughput increased by $4.1 \%$ for uncoded bit exchange and up to $11.6 \%$ for coded bit exchange. As a trade-off, the requirement on backhaul capacity between sites increases, too: $9 \mathrm{Mbps}$ (uncoded bit) and $900 \mathrm{Mbps}$ (5-bit soft values of coded bits) are required on average for the information exchange between BSs.

\section{REFERENCES}

[1] E. Dahlman, S. Parkvall, J. Skold, and P. Beming, 3G Evolution: HSPA and LTE for Mobile Broadband, 2nd ed. Academic Press, October 2008

[2] J. Andrews, "Interference cancellation for cellular systems: a contemporary overview," IEEE Wireless Communications, pp. 19-29, Apr 2005.

[3] S. Venkatesan, "Coordinating Base Stations for Greater Uplink Spectral Efficiency in a Cellular Network," in Proceedings of the 18th Annual IEEE International Symposium on Personal, Indoor and Mobile Radio Communications (PIMRC), 2007.

[4] P. Marsch and G. Fettweis, "A Framework for Optimizing the Uplink Performance of Distributed Antenna Systems under a Constrained Backhaul," in Proceedings of the IEEE International Conference on Communications (ICC), 2007.

[5] C. Hoymann, L. Falconetti, and R. Gupta, "Distributed Uplink Signal Processing of Cooperating Base Stations based on IQ Sample Exchange," in Proceedings of the IEEE ICC, 2009.

[6] P. Dent, B. Gudmudson, and M. Ewerbring, "CDMA-IC: A novel Code Division Multiple Access Scheme based on Interference Cancellation," in Proceedings of the IEEE Personal Indoor Mobile Radio Communication Conference, 1992, pp. 411-415.

[7] Next Generation Mobile Networks (NGMN) Alliance, "NGMN Radio Access Performance Evaluation Methodology," NGMN White Paper, January 2008.

[8] 3GPP, "3GPP TR 25.996, Spacial channel model for Multiple Input Multiple Output simulations," June 2007.

[9] K. Brueninghaus, D. Astely, T. Salzer, S. Visuri, A. Alexiou, S. Karger, and G. Seraji, "Link Performance Models for System Level Simulations of Broadband Radio Access Systems," IEEE 16th International Symposium on PIMRC, 2005. 